Pacific Journal of Mathematics

ON TIGHTEST PACKING IN THE MINKOWSKI PLAN

Hans SYlVAin Witsenhausen and Hans Zassenhaus 


\title{
ON TIGHTEST PACKINGS IN THE MINKOWSKI PLANE
}

\section{R. L. Graham, H. S. Witsenhausen and H. J. Zassenhaus}

\begin{abstract}
The J. H. Folkman and R. L. Graham 1969 method of obtaining a packing inequality for finite plane simplicial complexes that are admissible for Euclidean distance is generalized for arbitrary Minkowski distances, a slackness measure is defined and the $N$. Oler 1961 result concerning tightest admissible Jordan triangulations is extended to tightest finite packings.
\end{abstract}

1. Introduction. The classical problem of the geometry of numbers is concerned with the existence of optimal lattices in real affine $n$-space $\boldsymbol{R}^{n}$ meeting geometric conditions with regard to a Minkowski distance $d$. For example, the Minkowski packing problem asks for the existence of $n$-dimensional lattices $L$ of $\boldsymbol{R}^{n}$ of minimal mesh (critical lattices) such that the $d$-packing condition

$$
d(P, Q) \geqq 1
$$

is satisfied for any two distinct points $P, Q$ of $L$. Since the work of Thue on circle packings in the plane, many people have noted that the mesh of a lattice is the inverse of the density of its points and have asked whether there are arbitrary pointsets of maximal density satisfying the packing condition. C. A. Rogers [8] showed in 1951, for example, that for the Euclidean plane no packing is denser than the critical lattice.

The question which first was taken up by Thue concerns itself with the metric characteristics of finite pointsets of $R^{2}$ satisfying the Euclidean distance packing condition. In this paper we develop the motivation for giving the metric characterization in terms of invariant measures and we solve the Thue problem (which was solved for plane packings in Jordan polygons by N. Oler [3] in 1961) for the vertex set of arbitrary finite plane simplicial complexes meeting the $d$-packing condition (cf. Theorem 4), thereby extending the method used by Folkman and Graham [2] in the case of Euclidean distance.

It is interesting to observe that the plane packings of a given finite number of points of minimal slackness measure (cf. [9]) must be part of a critical 2-lattice if the unit ball defining $d$ is strictly convex. On the other hand there are infinite irregular packings of maximal density which are not subsets of a critical 2-lattice.

Of course, the corresponding questions can be considered in more than two dimensions. As a consequence of the work of Minkowski, it is clear what the invariant measures for convex bodies must be, 
although the extension to arbitrary simplicial complexes requires certain modifications.

The final sections of the paper indicate the possibility of extending the study of equations in combinatorial topology (which forms the beginning of algebraic topology) to the study of inequalities for simplicial complexes (which forms the beginning of metric topology).

\section{Affine Minkowski spaces.}

Definition. A linear Minkowski space is a finite-dimensional real Banach space. That is, it is a real linear space provided with a norm $x \rightarrow\|x\|$ such that (i) $\|\lambda x\|=|\lambda| \cdot\|x\|$ for real $\lambda$, (ii) $\|x+y\| \leqq$ $\|x\|+\|y\|$ and (iii) $\|x\|=0 \Rightarrow x=0$. Thus a norm is a convex, positively homogeneous function vanishing only at the origin.

The unit ball $\{x \mid\|x\| \leqq 1\}$ is a compact convex set symmetric about the origin and it characterizes the norm.

An affine Minkowski space is a metric space obtained from a real affine space by defining a distance $d(A, B)$ or $|A B|$ by $d(A, B)=\|\overrightarrow{A B}\|$ where (i) the vector $\overrightarrow{A B}$ is an element of the linear space of equivalence classes, modulo translations, of ordered pairs of points $(A, B)$ and (ii) $\|\cdot\|$ is a norm on that linear space.

In an affine Minkowski space there is a family of unit balls differing among themselves only by translations.

Convexity of distance. The convexity of the norm implies that the distance from a fixed point $F$ to an arbitrary point $P$ is a convex function of $P$, for $P$ varying over the affine Minkowski space or, $a$ fortiori, over a convex subset thereof, such as a straight line. This is a key tool of Minkowski geometry, used as follows. Let $[A, B]$ denote the line segment joining points $A$ and $B$, including these points, while $(A, B],[A, B)$ and $(A, B)$ denote the remainder of this set when $A, B$ or both are removed.

FACT 1:

(i) If $|P A| \leqq|P C|$ and $B \in[A, C]$ then $|P B| \leqq|P C|$.

(ii) If $|P A|<|P C|$ and $B \in[A, C)$ then $|P B|<|P C|$.

(iii) If $|P A| \leqq|P B|$ and $B \in[A, C]$ then $|P B| \leqq|P C|$.

(iv) If $|P A|<|P B|$ and $B \in[A, C)$ then $|P B|<|P C|$.

Some affine properties. In affine geometry two figures are called "similar and similarly situated" or "parallel" if they differ either by a translation or else by a homothety (alias: dilatation). A homothety of center $C$ and ratio $\lambda$ (real, nonzero) sends a point $P$ into the point $Q$ satisfying $\overrightarrow{C Q}=\lambda \overrightarrow{C P}$. In such figures corresponding pairs of 
points determine parallel segments.

Two triangles $A B C$ and $A^{\prime} B^{\prime} C^{\prime}$ with parallel bases $B C$ and $B^{\prime} C^{\prime}$ are said to have the "same altitude" when for any point $P$ on line $B C$ the fourth vertex $Q$ of parallelogram $P A A^{\prime} Q$ belongs to line $B^{\prime} C^{\prime}$.

FACT 2: The ratio of the areas of these triangles (an affine invariant) is the ratio of their bases, considered as proportional vectors and, a fortiori, of the lengths of their bases.

The case of the plane. Some properties of the affine Minkowski plane, valid for all norms, are needed for the sequel. Denote by $\Delta^{*}$ the infimum (which, by compactness, is actually a minimum) of the areas of all equilateral triangles with sides of unit length. The reference area with respect to which areas are measured is irrelevant since all properties ultimately refer to ratios of areas. In the Euclidean case, $\Delta^{*}$ is $\sqrt{3} / 4$ unit squares.

Lemma 1. The area $E$ of an equilateral triangle with sides of length $\lambda$ is not less than $\lambda^{2} \Delta^{*}$.

Proof. A homothety of ratio $\lambda^{-1}$ and arbitrary center maps the given triangle into a triangle with unit sides and area $\lambda^{-2} E$ which cannot, by definition, be less than $\Delta^{*}$.

The sets $\{P|| C P \mid=r\}$ for given $C$ and $r>0$ are called Minkowski circles of center $C$, radius $r$.

Lemma 2. If point $P$ is not located on line $\mathscr{L}$, then there exists at least one equilateral triangle $P Q R$ with $Q$ and $R$ on $\mathscr{L}$.

Proof. Let $A, B$ be distinct points on $\mathscr{L}$. By continuity, the Minkowski circles with centers $A, B$ and radii $|A B|$ have common points. Let $C$ be one of them. The triangle $A B C$ is equilateral. Draw parallels to $A C$ and to $B C$ through $P$. Together with $\mathscr{L}$ they form a triangle parallel to triangle $A B C$, hence, equilateral.

\section{A lower bound on the area of a triangle.}

For a triangle $P_{1} P_{2} P_{3}$ it may be assumed without loss that $s_{1}=$ $\left|P_{2} P_{3}\right| \geqq s_{2}=\left|P_{1} P_{3}\right| \geqq s_{3}=\left|P_{1} P_{2}\right|$. Then the three triangle inequalities are subsumed under

$$
s_{1} \leqq s_{2}+s_{3} .
$$

When $P_{1} P_{2} P_{3}$ are collinear the triangle is called degenerate and (1) holds with equality. The converse of this statement is only true in the special case where the unit ball is strictly convex. On the 
other hand, the area $A$ of the triangle vanishes if and only if it is degenerate. Thus if $s_{2}+s_{3}-s_{1}>0$ then $A>0$. In Minkowski geometry the lengths of the sides of a triangle do not determine its area but a lower bound can be obtained when (1) holds with strict inequality.

Theorem 1. The area $A$ of a triangle with side lengths $s_{1} \geqq s_{2} \geqq s_{3}$ is no less than $s_{2} \Delta^{*}\left(s_{2}+s_{3}-s_{1}\right)$.

Proof. Let $P_{1}, P_{2}, P_{3}$ be the vertices opposed to $s_{1}, s_{2}, s_{3}$, respectively. Construct (Lemma 2) an equilateral triangle $P_{2} R S$ with $R S$ on the line and in the direction $P_{1} P_{3}$. Let $E$ be its area and $\lambda$ its side length. Then $\lambda+s_{1} \geqq s_{2}+s_{3}$. For indeed interval [RS] cannot be contained in interval $\left(P_{1} P_{3}\right)$ because this would imply $s_{1}=\left|P_{2} P_{3}\right| \leqq$ $\left|P_{2} S\right|+\left|S P_{3}\right|=|R S|+\left|S P_{3}\right|=\left|R P_{3}\right|<\left|P_{1} P_{3}\right|=s_{2}$. If $P_{1}$ and $/$ or $P_{3}$ belong to $[R S]$ then convexity of the distance from $P_{2}$ to $R,\left(P_{1}\right.$ or $\left.P_{3}\right), S$ gives $s_{3} \leqq \lambda$ to which one need only add $s_{2} \leqq s_{1}$. If interval [RS] does not meet $\left[P_{1} P_{3}\right]$ then it cannot be on the side of $P_{3}$ by convexity of the distances from $P_{2}$, unless $s_{1}=s_{3}=\lambda$ whence $\lambda+s_{1}=$ $s_{2}+s_{3}$. With $R S$ on the side of $P_{1}$ one has $s_{3}=\left|P_{2} P_{1}\right| \leqq\left|P_{2} S\right|+$ $\left|S P_{1}\right|=|R S|+\left|S P_{1}\right|=\left|R P_{1}\right|$ so that $s_{2}+s_{3} \leqq\left|P_{1} P_{3}\right|+\left|R P_{1}\right|=\left|R P_{3}\right| \leqq$ $\left|R P_{2}\right|+\left|P_{2} P_{3}\right|=\lambda+s_{1}$.

The triangles $P_{2} R S$ and $P_{2} P_{1} P_{3}$ have a common altitude so that $A=s_{2} E / \lambda$. By Lemma $1, A \geqq s_{2} \Delta^{*} \lambda \geqq s_{2} \Delta^{*}\left(s_{2}+s_{3}-s_{1}\right)$.

\section{Classification of angles.}

Definition. A proper angle of vertex $P$ is a closed convex set bounded by two half lines, carried by distinct lines, with common endpoint $P$.

When the two half lines merge into a single half line one has the, improper, zero angle. When the convex set becomes a half-plane one has the, improper, straight angle.

Definition. Two angles are supplementary when their intersection is a zero angle and their union a straight angle.

Thus any angle has two supplements differing only by reflection in the common vertex. The supplements of a proper angle are proper angles.

One way to discuss angles quantitatively is to study the distances between points at unit distance from the apex on the two sides of the angle and its supplement.

Consider angle $X O Y$ where $|O X|=|O Y|=1$. Let $Z$ be symmetric to $X$ with respect to $O$, so that $|O Z|=1$. 
Definitions. The angle $X O Y$ will be called

$\begin{array}{llll}\text { narrow } & \text { when } & |X Y|<1 \\ \text { wide } & \text { when } & |Y Z|<1 \\ \text { intermediate } & \text { when } & |X Y| \geqq 1 \\ \text { critical } & \text { when } & |X Y|=1 .\end{array}$ and $\quad|Y Z| \geqq 1$

By the triangle inequality $|X Y|+|Y Z| \geqq 2$, hence, an angle cannot be both wide and narrow, though $|X Y|=|Y Z|=1$ is possible (take the unit ball to be a parallelogram).

The classification of angles as narrow, wide and intermediate is, therefore, exhaustive and mutually exclusive.

\section{Properties of angles.}

Lemma 3. An angle contained in a narrow angle is narrow.

Proof. It suffices to consider the case where the angles have a common side. Let angle $Y O Z$ be contained in narrow angle $X O Y$ with $|O X|=|O Y|=|O Z|=1,|X Y|<1$. Construct the parallelograms $O Y X R$ and $O Y Z U$. Line $O Z$ meets $X Y$ at $Q$ and $R X$ at $S$. Line $Z U$ meets $X Y$ at $V$. Convexity of the distances from $O$ to $X, Q, Y$ implies $|O Q| \leqq 1$. Convexity of the distances from $O$ to $S, X, R$ implies $|O S|>1$. Hence, $Z$ belongs to the intervals $[Q, S)$ and $[V, U)$, $V$ belongs to interval $(X, Y)$. Convexity of the distance of $Y$ to $V, Z, U$ when $|Y V|<|Y X|<1$ and $|Y U|=|O Z|=1$ implies $|Y Z|<$ 1. Thus, angle $Y O Z$ is narrow as claimed.

If in the above argument one had $|X Y| \leqq 1$, then $Z$ is shown to belong to $[Q, S]$ so that $|Y V| \leqq|Y X| \leqq 1$ and one concludes $|Y Z| \leqq 1$. This may be stated as

Lemma 3'. An angle contained in a narrow or critical angle is itself narrow or critical.

Another crucial fact is

Lemma 4. In a triangle, a side opposed to a narrow angle is strictly shorter than one of the other sides.

Proof. Let $A B C$ have a narrow angle at $B$. Construct $D$ on the half line from $B$ through $C$ so that $|B D|=|A B|$. By assumption $|A D|<|A B|$. If $C$ belongs to interval $[B, D]$ then the distances from $A$ are in order $|A B|,|A C|,|A D|<|A B|$ so that $|A C|<|A B|$ by convexity. If $D$ is between $B$ and $C$ then $|A C| \leqq|A D|+|D C|<|B D|+|D C|=$ $|B D|$. 
LEMMA 5. If $X O Y$ is an intermediate angle then there exists an equilateral triangle $Y U V$ with $U, V$ on line $O X$ and $O$ contained in segment $[U, V]$.

Proof. One may assume $|O X|=|O W|=|O Z|=1$ with $W$ on half line $O Y, Z$ symmetric to $X$ with respect to $O$. Then $|X W| \geqq$ $1,|W Z| \geqq 1$. Construct equilateral triangle $X R O$ with $R$ on the same side of line $O X$ as $W$. A translation of $X R O$ by $\overrightarrow{O Z}$ gives $O S Z$. If $Y$ is located in angle $R O S$, line $O Y$ meets interval $(R S)$ at $P$. Translate triangle $X R O$ by $\overrightarrow{R P}$ then perform a homothety of center $O$ to bring $P$ onto $Y$, obtaining the desired triangle. If $W$ is located in angle $X O R$ then $|X W|=1$ by Lemma $3^{\prime}$ and $X O W$, an equilateral triangle, yields the desired triangle, by homothety. A similar argument applies if $W$ is located in angle SOZ.

LEMma 6. If triangle $A B C$ has an intermediate angle at $A$, then its area $\alpha$ is no less than $\Delta^{*}|A B| \cdot|A C|$.

Proof. By Lemma 5 there exists an equilateral triangle $B R S$ with $R S$ on line $A C$ and $A \in[R, S]$. Let $\lambda$ be the length of its sides and $E$ its area. By convexity of the distances from $B$ one has $|A B| \leqq$ $\lambda$. Since triangles $B A C$ and $B R S$ have a common altitude one has, using Lemma $1, \alpha=E \cdot|A C| / \lambda \geqq \Delta^{*}|A C| \lambda \geqq \Delta^{*}|A C||A B|$.

\section{A bound on the area of certain quadrilaterals.}

THEOREM 2. In a convex quadrilateral in which any diagonal is at least as long as any side, the area is no less than $\Delta^{*}\left(s t+s^{\prime} t^{\prime}\right)$, where $s, t$ are the lengths of an appropriate pair of adjacent sides and $s^{\prime}, t^{\prime}$ are the lengths of the two other sides.

Proof. If any of the four angles of convex quadrilateral $A B C D$ were narrow, then the diagonal opposite to this angle would, by Lemma 4 , be strictly shorter than one of the sides, contradicting the hypothesis. Assume two adjacent angles say, $A, B$, are both wide. Assume without loss of generality that $D$ is at least as close as $C$ to line $A B$. Draw $C X$ parallel to and oriented as $B A$. Then, angle $B C X$, parallel to the supplement of the wide angle at $B$, would be narrow. By Lemma 3, angle $C$ would also be narrow, a contradiction. Thus there is a pair of opposite angles that are both intermediate. Applying Lemma 6 to the corresponding triangles establishes the claim.

\section{Invariant measures on plane simplicial complexes.}

DEFinition. A collection $\mathscr{P}$ of points, straight segments and 
solid triangles is called a finite plane simplicial complex (FPSC) if:

1. The number $a_{0}(\mathscr{S})$ of points in $\mathscr{S}$ ("vertices" or "0-faces" of $\mathscr{S}$ ) is finite;

2. Every straight segment in $S$ ("edge" or "1-face" of $\mathscr{S}$ ) is the closed straight segment $[P Q]$ connecting two distinct vertices $P, Q$ of $\mathscr{S}$ such that no vertex of $\mathscr{S}$ is between $P$ and $Q$; the intersection of two distinct edges of $\mathscr{S}$ either is empty or it is a vertex of $\mathscr{S}$;

3. Every solid triangle in $\mathscr{S}$ ("2-face" of $\mathscr{S}$ ) is the convex hull $[P Q R]$ of some set of three distinct vertices $P, Q, R$ of $\mathscr{S}$ such that the edges $[P Q],[Q R],[R P]$ of $[P Q R]$ are edges of $\mathscr{S}$ and that $P, Q, R$ are the only vertices of $\mathscr{S}$ contained in $[P Q R]$.

It follows that the number $a_{1}(\mathscr{S})$ of edges of $\mathscr{S}$ and the number $a_{2}(\mathscr{S})$ of 2 -faces of $\mathscr{S}$ are finite.

If $[P Q R]$ is a 2 -face of $\mathscr{S}$ then $P, Q, R$ are noncollinear.

The intersection of two 2 -faces of $\mathscr{S}$ either is empty or it is a common vertex or it is a common edge. In the latter event the vertices which are not in common lie on opposite sides of the straight line generated by the common edge of the two solid triangles.

An edge of $\mathscr{S}$ either is an edge of 2,1 or 0 solid triangles of $\mathscr{S}$. In the first case it is an inner edge, in the second and third cases it is a boundary edge; in the second case it is a bounding edge, in the third case it is a nonbounding edge.

A vertex of $\mathscr{S}$ either is a boundary vertex (i.e., a vertex of a boundary edge of $\mathscr{S}$ or an isolated vertex) or it is an inner vertex. The boundary edges and vertices of $\mathscr{S}$ form an FPSC which shall be denoted by $\partial \mathscr{S}$ and which shall be called the boundary of $\mathscr{S}$.

Definition. The pointset $|\mathscr{S}|$ formed by the set-theoretic union of the 0 -, 1 - and 2 -faces of the finite plane simplicial complex $\mathscr{S}$ is called the support of $\mathscr{S}$.

The support pointset also are called triangulable (plane) pointsets. The support of $\partial \mathscr{S}$ is easily seen to be the boundary of $|\mathscr{S}|$.

Examples of FPSC's are:

I. The empty FPSC; it will be denoted by $\varnothing$ and it is characterized by the numerical relations

$$
a_{0}(\varnothing)=a_{1}(\varnothing)=a_{2}(\varnothing)=0 ;
$$

II. The basic 0-complexes formed by just one vertex $P$; they are denoted by $\{P\}$; these $F P S C^{\prime}$ s are characterized by the numerical relation

$$
a_{0}(\mathscr{S})=1, \quad a_{1}(\mathscr{S})=a_{2}(\mathscr{S})=0 ;
$$


III. The basic 1-complexes formed by two distinct vertices $P, Q$ and the straight segment $[P Q]$ joining $P, Q$; it is denoted by $\{P Q\}$; the FPSC's obtained in this may are characterized by the numerical relations

$$
a_{0}(\mathscr{S})=2, \quad a_{1}(\mathscr{S})=1, \quad a_{2}(\mathscr{S})=0 ;
$$

IV. The FPSC formed by three noncollinear vertices $P, Q, R$, the straight segments $[P Q],[Q R],[R P]$ joining them and the solid triangle $[P Q R]$ generated by them; it is denoted by $\{P Q R\}$; the $F P S C^{\prime}$ s obtained in this way are called the basic 2-complexes and they are charaterized by

$$
a_{0}(\mathscr{S})=3, \quad a_{1}(\mathscr{S})=3, \quad a_{2}(\mathscr{S})=1 .
$$

LEMma 7. The union and the intersection of two triangulable plane pointsets are triangulable.

Proof. One has to show that for any two FPSC's $\mathscr{S}_{1}, \mathscr{S}_{2}$ there are $F P S C$ 's $\mathscr{C}, \mathscr{T}$ such that

$$
|\mathscr{U}|=\left|\mathscr{S}_{1}\right| \cup\left|\mathscr{S}_{2}\right|, \quad|\mathscr{T}|=\left|\mathscr{S}_{1}\right| \cap\left|\mathscr{S}_{2}\right| \cdot
$$

This is clear if $\mathscr{S}_{1}$ and $\mathscr{S}_{2}$ are compatible in the sense that the union of $\mathscr{S}_{1}$ and of $\mathscr{S}_{2}$ is a FPSC. In that case we set

$$
\mathscr{C}=\mathscr{S}_{1} \cup \mathscr{S}_{2}, \quad \mathscr{T}=\mathscr{S}_{1} \cap \mathscr{S}_{2} \text {. }
$$

In general, for the the purpose of constructing $\mathscr{H}, \mathscr{T}$, we will have to modify $\mathscr{S}_{i}$ into another FPSC $\mathscr{S}_{i}^{\prime}$ with the same support as $\mathscr{S}_{i}(i=1,2)$ in such a way that $\mathscr{S}_{1}^{\prime}, \mathscr{S}_{2}^{\prime}$ are compatible.

This will be done by mutual refinement of $\mathscr{S}_{1}^{\prime}, \mathscr{S}_{2}^{\prime}$.

An elementary refinement of $\mathscr{S}_{1}$ is defined as the replacement of $\mathscr{S}_{1}$ by one of the following FPSC's:

(a) exchange of $[P Q]$ for $X,[P X],[X Q]$ and a new vertex $X$, retaining the other items of $\mathscr{S}_{1}$ provided $X$ is between $P, Q$ and $[P Q]$ is a nonbounding boundary edge of $\mathscr{S}_{1}$;

(b) exchange of $[P Q],[P Q R]$ for $[P X],[X Q],[X R],[P X R],[Q X R]$ and a new vertex $X$, retaining the other items of $\mathscr{S}_{1}$ provided $X$ is between $P, Q$ and $[P Q]$ is a boundary edge of $\mathscr{S}_{1}$;

(c) exchange of $[P Q],[P Q R],[P Q S]$, for $[P X],[X Q],[X R],[X S]$, $[P X R],[Q X R],[P X S],[Q X S]$ and a new vertex $X$ provided $X$ is between $P, Q$ and $[P Q[$ is an inner edge;

(d) exchange of $[P Q R]$ for $[P X],[Q X],[R X],[P Q X],[Q R X]$, $[R P X]$ and a new vertex $X$, retaining the other items of $\mathscr{S}_{1}$ provided $X$ is in the interior of the 2 -face $[P Q R]$ of $\mathscr{S}_{1}$.

In any case one obtains a new FPSC with the same support as $\mathscr{S}_{1}$. Any FPSC obtained by a chain of elementary refinements from 
$\mathscr{S}_{1}$ is a refinement of $\mathscr{S}_{1}$.

Utilizing for the purpose of refining $\mathscr{S}_{i}$ both the vertices of $\mathscr{S}_{3-i}$ as well as the points which are obtained by intersecting an edge of $\mathscr{S}_{i}$ with a nonparallel edge of $\mathscr{S}_{3-i}$ we obtain compatible refinements $\mathscr{S}_{i}^{\prime}$ of $\mathscr{S}_{i}$ for $i=1,2$ !

Definition. A real valued function $f$ of the $F P S C^{\prime} \mathrm{s}$ is called additive if it satisfies the conditions:

(1) $f$ depends only on the support of its argument;

(2) if $\mathscr{S}_{1}, \mathscr{S}_{2}$ are compatible then

$$
f\left(\mathscr{S}_{1}\right)+f\left(\mathscr{S}_{2}\right)=f\left(\mathscr{S}_{1} \cup \mathscr{S}_{2}\right)+f\left(\mathscr{S}_{1} \cap \mathscr{S}_{2}\right) \text {. }
$$

Using the terminology introduced in the proof of Lemma 7 it is clear that condition (1) is satisfied if and only if $f$ does not change under elementary refinement.

Furthermore, any real valued function $f$ of the $F P S C^{\prime}$ s satisfying (1) induces a real valued function $\bar{f}$ on the triangulable (plane) pointsets by setting

$$
\bar{f}(|\mathscr{S}|)=f(\mathscr{S}) \text {. }
$$

The condition (2) is tantamount to the condition

$$
\bar{f}\left(S_{1}\right)+\bar{f}\left(S_{2}\right)=\bar{f}\left(S_{1} \cup S_{2}\right)+\bar{f}\left(S_{1} \cap S_{2}\right)
$$

for the function $\bar{f}$, to be satisfied by all pairs of triangulable plane pointsets $\mathscr{S}_{1}, \mathscr{S}_{2}$.

Examples are:

I. The 1-function

$$
1(\mathscr{S})=1
$$

II. The area function

$$
\mathscr{A}(\mathscr{S})=\sum_{[P Q R] \in \mathscr{O}} \mathscr{A}_{[P Q R]}
$$

III. The perimeter function

$$
\mathscr{P}(\mathscr{S})=\sum_{[P(\ell) \in \partial}|P Q|+\sum_{\substack{[P Q] \in \mathcal{C} \\[P Q] \text { nonbounding }}}|P Q| ;
$$

IV. The Euler-Poincaré characteristic

$$
\chi(\mathscr{S})=a_{0}(\mathscr{S})-a_{1}(\mathscr{S})+a_{2}(\mathscr{S}) .
$$

It is not difficult to verify in each case the invariance under elementary refinement and the additivity condition.

The additive functions form a linear space over the real number field $\mathscr{R}$. For the four FPSC's $\varnothing$, 


$$
\begin{aligned}
& \mathscr{S}_{0}=\{P\}, \quad(P \text { some point }), \\
& \mathscr{S}_{1}=\{P Q\}, \quad(P, Q \text { points of distance } 1), \\
& \mathscr{S}_{2}=\{P Q R\}, \quad([P Q R] \text { an equilateral triangle of side length } 1 \text { and } \\
& \left.\quad \text { of area } \Delta^{*}\right),
\end{aligned}
$$

we obtain the following table of values:

\begin{tabular}{c|cccc} 
& 1 & $\chi$ & $\mathscr{P}$ & $\mathscr{A}$ \\
\hline$\varnothing$ & 1 & 0 & 0 & 0 \\
$\mathscr{S}_{0}$ & 1 & 1 & 0 & 0 \\
$\mathscr{S}_{1}$ & 1 & 1 & 1 & 0 \\
$\mathscr{S}_{2}$ & 1 & 1 & 3 & $\Delta^{*}$ \\
.
\end{tabular}

Noting that

$$
\Delta^{*}>0
$$

it follows that the 4 functions $1, \chi, \mathscr{P}, \mathscr{A}$ are linearly independent. They have the following additional properties:

(3) Invariance under translation;

(4) The function restricts to a linear function of $|P Q|$ for basic 1-complexes $[P Q]$;

(5) Continuity. ${ }^{1}$

Definition. Real valued functions of the FPSC's satisfying the conditions (1)-(5) are called invariant measures.

Theorem 3. The invariant measures form a linear space over $\mathscr{R}$ spanned by $1, \chi, \mathscr{P}, \mathscr{C}^{2}$

We have pointed out already that $1, \chi, \mathscr{P}, \mathscr{A}$ are linearly independent invariant measures.

Let $f$ an invariant measure.

In order to see that $f$ is an $\mathscr{R}$-linear combination of $1, \gamma, \mathscr{P}, \mathscr{A}$ it suffices to show that the difference $f-f(\varnothing) \cdot 1$ is an $\mathscr{R}$-linear

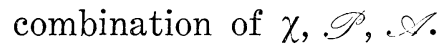

${ }^{1}$ A sequence $\left(\mathscr{S}_{n}\right)$ of $F P S C^{\prime}$ s is said to be convergent towards the FPSC.Y if for some index $N$ we have the equations $a_{0}\left(\mathscr{C}_{n}\right)=a_{0}\left(\mathscr{S}^{\rho}\right)$ for all $n>N$ and if, moreover for $n>N$ the vertices of $\mathscr{S}_{n}$ can be so numbered that they form $a_{0}(\mathscr{S})$ pointsequences $\left(P_{i n}\right)$ converging towards the vertices $P_{i}$ of $\mathscr{P}\left(1 \leqq i \leqq a_{0}(\mathscr{S})\right)$ and if, finally, the edges $\left[P_{i n} P_{j n}\right]$ or the 2 -faces $\left[P_{i n} P_{j n} P_{k n}\right]$ belong to $\mathscr{S}_{n}$ if and only if $\left[P_{i} P_{j}\right]$ or $\left[P_{\imath} P_{j} P_{k}\right]$ belong to $\mathscr{P}$.

The continuity of the additive function $f$ requires that $\lim _{n \rightarrow \infty} f\left(\mathscr{S}_{n}\right)=f(\mathscr{S})$ whenever the sequence $\left(\mathscr{S}_{n}\right)$ converges to $\mathscr{S}$.

${ }^{2}$ Compare Blaschke [1]. 
In view of the remark that every $F P S C$ is the union of finitely many compatible basic FPSC's it suffices, because of (2), to show that the restriction of an invariant measure vanishing on $\varnothing$ to the basic $F P S C^{\prime}$ s is an $\mathscr{R}$-linear combination of the restriction of $\chi, \mathscr{P}, \mathscr{A}$.

We note that $\chi$ restricts to 1 on the nonempty basic FPSC's.

Due to the translation invariance of $f$ there exists a real number $\lambda_{1}$ such that $f+\lambda_{1} \chi$ restricts to zero on the basic $0-F P S C^{\prime}$ 's. Without loss of generality we assume that already $f$ itself restricts to zero on the basic $0-F P S C^{\prime} \mathrm{s}$.

By (4) there exists a real number $\lambda_{2}$ such that $f+\lambda_{2} \mathscr{P}$ restricts to zero on the basic $1-F P S C^{\prime}$ s. Without loss of generality we assume that already $f$ itself restricts to zero on the basic 0 - or $1-F P S C^{\prime}$ s. Hence (2) assumes the stronger form

$$
f\left(\mathscr{S}_{1} \cup \mathscr{S}_{2}\right)=f\left(\mathscr{S}_{1}\right)+f\left(\mathscr{S}_{2}\right)
$$

in the event that $\mathscr{S}_{1}, \mathscr{S}_{2}$ are $F P S C^{\prime}$ s which have no 2 -face in common.

We apply this strict additivity and the translation invariance of invariant measures and the continuity property to the rectangular simplicial complexes

$$
\begin{aligned}
\mathscr{S}\left(x_{0}, y_{0}, \alpha, \beta\right)= & \left\{\left(x_{0}, y_{0}\right),\left(x_{0}+\alpha, y_{0}\right),\left(x_{0}, y_{0}+\beta\right),\left(x_{0}+\alpha, y_{0}+\beta\right),\right. \\
& {\left[\left(x_{0}, y_{0}\right)\left(x_{0}+\alpha, y_{0}\right)\right],\left[\left(x_{0}+\alpha, y_{0}\right)\left(x_{0}+\alpha, y_{0}+\beta\right)\right], } \\
& {\left[\left(x_{0}+\alpha, y_{0}+\beta\right)\left(x_{0}, y_{0}+\beta\right)\right],\left[\left(x_{0}, y_{0}+\beta\right)\left(x_{0}, y_{0}\right)\right], } \\
& {\left[\left(x_{0}, y_{0}\right)\left(x_{0}+\alpha, y_{0}+\beta\right)\right], } \\
& {\left.\left[x_{0}, y_{0}\right)\left(x_{0}+\alpha, y_{0}\right)\left(x_{0}+\alpha, y_{0}+\beta\right)\right], } \\
& {\left.\left[\left(x_{0}, y_{0}\right)\left(x_{0}, y_{0}+\beta\right)\left(x_{0}+\alpha, y_{0}+\beta\right)\right]\right\} }
\end{aligned}
$$

where

$$
x_{0}, y_{0}, \alpha, \beta \in \mathscr{R}, \quad \alpha>0, \quad \beta>0,
$$

and obtain by application of the usual Euclid-Riemann-Jordan area theory that

$$
\begin{aligned}
f\left(\mathscr{S}\left(x_{0}, y_{0}, \alpha, \beta\right)\right) & =\alpha \beta f(\mathscr{S}(0,0,1,1)) \\
& =f(\mathscr{S}(0,0,1,1)) \mathscr{A}\left(\mathscr{S}\left(x_{0}, y_{0}, \alpha, \beta\right)\right) .
\end{aligned}
$$

Hence,

$$
f=f(\mathscr{P}(0,0,1,1)) \mathscr{A}
$$

and the theorem is proved.

CoRollary. The invariant measures $\chi, \mathscr{P}, \mathscr{A}$ are characterized, up to proportionality, by the two properties that: 
(a) They vanish on the empty set;

(b) Under the application of a homothety in the ratio $\lambda: 1$ their value is multiplied by $1,|\lambda|, \lambda^{2}$ respectively.

In this way dimensionality enters invariant measure theory.

\section{A packing inequality.}

Definition. The finite plane simplicial complex $\mathscr{S}$ provides a finite packing (for the given Minkowski distance function) if the distance between any two distinct vertices is not smaller than 1 in case the straight segment joining them belongs to the support of $\mathscr{S}$.

Examples are provided by the FPSC with vertex set contained in an admissible 2-lattice (see [7] for definitions).

Definition. An inequality of the form

$$
a_{0}(\mathscr{S}) \leqq f(\mathscr{S})
$$

which is satisfied by the invariant measure $f$ for all finite packings $\mathscr{S}$ is said to be a packing inequality.

Definition. The packing inequality

$$
a_{0}(\mathscr{S}) \leqq f_{1}(\mathscr{S})
$$

is said to be better than the packing inequality

$$
a_{0}(\mathscr{S}) \leqq f_{2}(\mathscr{S})
$$

if $f_{1} \neq f_{2}$ and if $f_{2}-f_{1}$ is nonnegative for all $F P S C^{\prime}$ s.

We note that the relation "better than" is nonreflexive, but transitive; in other words, it establishes a partial ordering on the packing inequalities. Therefore it makes sense to ask the question: Is there a best packing inequality? Of course, if it exists at all it will be unique.

The existence of a best packing inequality, conjectured earlier by one of the authors, was proven for Jordan triangulations by N. Oler [3] (1961) and for finite packings that are admissible for the Eucliden distance function, using another method, by Folkman and Graham [2] (1969). ${ }^{3}$ The method used by the latter two authors is generalized here in order to show:

Theorem 4. For every Minkowski distance $d$ there is the following best packing inequality:

$$
a_{0}(\mathscr{S}) \leqq \frac{\mathscr{A}(\mathscr{S})}{2 \Delta^{*}}+\frac{\mathscr{P}(\mathscr{S})}{2}+\chi(\mathscr{S}) .
$$

${ }^{3}$ The "best" property was not shown explicitly. 
Proof. Let us begin with the proof of the optimum property of (3).

For this purpose we must show that any packing inequality

$$
a_{0}(\mathscr{S}) \leqq f(\mathscr{S})
$$

in which

$$
f=\lambda_{0} \cdot 1+\lambda_{1} \chi+\lambda_{2} \mathscr{P}+\lambda_{3} \mathscr{A}
$$

implies the inequalities

$$
\begin{aligned}
& \lambda_{0} \geqq 0, \\
& \lambda_{1} \geqq 1, \\
& \lambda_{2} \geqq \frac{1}{2}, \\
& \lambda_{3} \geqq 1 /\left(2 \Delta^{*}\right) .
\end{aligned}
$$

Suppose $\mathscr{S}$ is a finite packing. There is a translation $\tau$ such that the supports of the image packings $\tau^{i} \mathscr{S}$ are disjoint. Hence we have the finite packings $\mathscr{S} \cup \tau(\mathscr{S}) \cup \cdots \cup \tau^{n-1}(\mathscr{S})$ for all of which (4) is valid so that the inequalities

$$
n a_{0}(\mathscr{S}) \leqq \lambda_{0}+n \lambda_{1} \chi(\mathscr{S})+n \lambda_{2} \mathscr{P}(\mathscr{S})+n \lambda_{3} \mathscr{A}(\mathscr{S})
$$

or

$$
a_{0}(\mathscr{S}) \leqq \lambda_{0} / n+\lambda_{1} \chi(\mathscr{S})+\lambda_{2} \mathscr{P}(\mathscr{S})+\lambda_{3} \mathscr{A}(\mathscr{S})
$$

for $n=1,2, \cdots$ are obtained. Hence

$$
a_{0}(\mathscr{S}) \leqq \lambda_{1} \chi(\mathscr{S})+\lambda_{2} \mathscr{P}(\mathscr{S})+\lambda_{3} \mathscr{A}(\mathscr{S})
$$

and by substitution of $\{P\}$ for $\mathscr{S},(6)$ yields $(5 b)$. Furthermore, upon substitution of $Q$ for $\mathscr{S}$ in (4) we obtain (5a).

Choose two points $P, Q$ with $d(P, Q)=1$. Let $P_{0}=P, P_{1}=Q$, and choose $P_{i}$ on the straight line joining $P$ and $Q$ so that $d\left(P_{i}, P_{j}\right)=$ $|i-j|, i, j=2,3, \cdots$. Let $\mathscr{S}(P, Q, n)$ be the finite packing that is provided by the vertices $P_{0}, \cdots, P_{n}$ together with the straight segments $\left[P_{0} P_{1}\right], \cdots,\left[P_{n-1} P_{n}\right]$. Upon substitution of $\mathscr{S}(P, Q, n)$ in (6) we obtain the inequalities

$$
n+1 \leqq \lambda_{1}+2 \lambda_{2} n
$$

or

$$
\lambda_{2} \geqq \frac{1}{2}+\left(1-\lambda_{1}\right) / 2 n
$$

for $n=1,2, \cdots$; hence $(5 \mathrm{c})$ holds. 
Finally let $[P Q R]$ be an equilateral triangle of sidelength 1 and of area $\Delta^{*}$ and let $\mathscr{S}(P, Q, R, n)$ be the finite packing provided by the $\left(\begin{array}{c}n+1 \\ 2\end{array}\right)$ vertices $P_{i k}$ which are obtained by setting

$$
\overrightarrow{P P_{i k}}=i \overrightarrow{P Q}+k \overrightarrow{P R} \quad(0 \leqq i, 0 \leqq k, i+k \leqq n)
$$

as well as the straight segments $\left[P_{i k} P_{i, k+1}\right](0 \leqq i, 0 \leqq k \leqq n-1-i)$ and $\left[P_{i k} P_{i+1, k}\right](0 \leqq i \leqq n-1-k, 0 \leqq k)$ and the $n^{2}$ triangles $\left[P_{i k} P_{i, k+1} P_{i+1, k}\right]$ $(0 \leqq i \leqq n-1-k, 0 \leqq k)$ and $\left[P_{i k} P_{i+1, k} P_{i, k-1}\right](0 \leqq i \leqq n-1-k, 1 \leqq k)$. Upon substitution of $\mathscr{S}(P, Q, R, n)$ in (6) we obtain the inequalities

$$
\left(\begin{array}{c}
n+1 \\
2
\end{array}\right) \leqq \lambda_{1}+3 \lambda_{2} n+\lambda_{3} \Delta^{*} / n^{2}
$$

or

$$
\lambda_{3} \geqq\left(1+\frac{1}{n}\right) / 2 \Delta^{*}-\lambda_{1} / n^{2} \Delta^{*}-3 \lambda_{2} / n \Delta^{*}
$$

for $n=1,2, \cdots$, hence (5d) holds.

Assume (3) does not hold. Then there must exist a finite packing with minimum value of $a(\mathscr{S})=a_{0}(\mathscr{S})+a_{1}(\mathscr{S})+a_{2}(\mathscr{S})$ for which (3) fails.

If $a_{0}(\mathscr{S})=0$ then both sides of (3) would vanish. Hence $a_{0}(\mathscr{S})>$ 0 . Also, if $a_{0}(\mathscr{S})=1$ then $a_{1}(\mathscr{S})=a_{2}(\mathscr{S})=0, \chi(\mathscr{S})=1$ and (3) would hold. Thus, we must have $a_{0}(\mathscr{S})>1$.

If $\mathscr{S}$ is the union of two compatible nonempty $F P S C^{\prime} \mathrm{s} \mathscr{S}_{1}, \mathscr{S}_{2}$ with empty intersection then we would find that $a\left(\mathscr{S}_{i}\right)<a(\mathscr{S})$ for $i=1,2$ so that (3) would hold for both $\mathscr{S}_{1}, \mathscr{S}_{2}$. Since the four functions $a_{s}, \mathscr{C}, \mathscr{P}, \chi$ are strictly additive again (3) would obtain. Hence we can assume $\mathscr{S}$ has only one connected component. This is the same as saying that the support of $\mathscr{S}$ is a connected pointset.

If there is a nonbounding edge $[P Q]$ of $\mathscr{S}$ then let us consider the finite packing $\mathscr{S}_{1}$ which is obtained by its deletion. Since $a_{0}\left(\mathscr{S}_{1}\right)=$ $a_{0}(\mathscr{S}), a_{1}\left(\mathscr{S}_{1}\right)=a_{1}(\mathscr{S})-1, a_{2}\left(\mathscr{S}_{1}\right)=a_{2}(\mathscr{S})$ it follows that $a\left(\mathscr{S}_{1}\right)<a(\mathscr{S})$ and therefore (3) is satisfied for $\mathscr{S}_{1}$. Moreover the right hand side of (3) for $\mathscr{S}$ is obtained by the addition of $(1 / 2) \cdot 2|P Q|-1$ to the corresponding expression for $\mathscr{S}_{1}$. Since this term is nonnegative by assumption a contradiction arises. Thus it follows that every edge of $\mathscr{S}$ is bounding.

Among the edges of $\mathscr{S}$ there is one of maximum length $\mu$, say the edge $[P Q]$. We make the further stipulation that the counterexample $\mathscr{S}$ is chosen among the finitely many ones with the same vertex set in such a way that the maximum length $\mu$ is as small as possible. Furthermore assume the number of maximum length edges 
is minimal.

There are two cases.

I. $[P Q]$ is a boundary edge. In this case $[P Q]$ bounds precisely one 2 -face of $\mathscr{S}$, say $[P Q R]$, and $[P Q]$ is the largest side of the triangle $[P Q R]$. Hence by Theorem 1

$$
\mathscr{A}(P Q R) \geqq(|P R|+|R Q|-|P Q|)(\max (|P R|,|R Q|)) \Delta^{*}
$$

and by the packing assumption $(|P R|,|R Q| \geqq 1)$ we have

$$
\mathscr{A}(P Q R) / 2 \Delta^{*}-(|P R|+|R Q|-|P Q|) / 2 \geqq 0 .
$$

Upon deletion of $[P Q]$ and $[P Q R]$ from $\mathscr{S}$ we obtain a finite packing $\mathscr{S}_{1}$ for which $a_{0}\left(\mathscr{S}_{1}\right)=a_{0}(\mathscr{S}), a\left(\mathscr{S}_{1}\right)<a(\mathscr{S}), \chi\left(\mathscr{S}_{1}\right)=\chi(\mathscr{S})$, $\mathscr{A}\left(\mathscr{S}_{1}\right)=\mathscr{A}(\mathscr{S})-\mathscr{A}(P Q R)$ and $P\left(\mathscr{S}_{1}\right)=P(\mathscr{S})-|P Q|+|P R|+$ $|R Q|$. By the induction hypothesis since $a_{1}(\mathscr{S})$ was assumed minimal, we see that (3) must hold for $\mathscr{S}_{1}$. Thus

$$
\begin{aligned}
a_{0}(\mathscr{S})=a_{0}\left(\mathscr{S}_{1}\right) \leqq & \frac{\mathscr{A}\left(\mathscr{S}_{1}\right)}{2 \Delta^{*}}+\frac{\mathscr{P}\left(\mathscr{S}_{1}\right)}{2}+\chi\left(\mathscr{S}_{1}\right) \\
= & \frac{\mathscr{A}(\mathscr{S})}{2 \Delta^{*}}+\frac{\mathscr{P}(\mathscr{S})}{2}+\chi(\mathscr{S}) \\
& -\left(\frac{\mathscr{A}(P Q R)}{2 \Delta^{*}}-(|P R|+|R Q|-|P Q|) / 2\right) \\
& \leqq \\
& \frac{\mathscr{A}(\mathscr{S})}{2 \Delta^{*}}+\frac{\mathscr{P}(\mathscr{S})}{2}+\chi(\mathscr{S})
\end{aligned}
$$

which contradicts the assumption that (3) fails for $\mathscr{S}$

II. The edge $[P Q]$ is an interior edge of $\mathscr{S}$. Suppose it is the edge which is common to the 2-faces $[P Q R]$ and $[P Q S]$ of $\mathscr{S}$. Here $[P Q]$ is the largest side of both triangles; $R$ and $S$ lie on opposite sides of the straight line connecting $P$ and $Q$.

If $|R S|<|P Q|$ then we could replace $\mathscr{S}$ by the union of the compatible finite packings $\mathscr{S}_{1}$ and $\{P, Q, R, S,[P R],[R Q],[Q S],[Q P]$, $[R S],[P R S],[R S Q]\}$ without changing any of the values of $\alpha_{0}, \chi, \mathscr{P}, \mathscr{A}$. But in the new counterexample the maximum length of the edges has not changed though the number of maximum length edges has decreased by 1 . Since this is contrary to previous assumptions concerning $\mathscr{S}$ we conclude that $|R S| \geqq|P Q|$. In this case it is not difficult to see that the quadrilateral $P R Q S$ is convex. By Lemma 4 none of the angles $P R Q, R Q S, Q S P, S P R$ is narrow. However, if two adjacent angles in $P R Q S$ were both wide, then arguing as in the proof of Theorem 2 we see that one of the interior angles must be narrow, 
again a contradiction. Thus, some pair of opposite angles of $P R Q S$ must both be intermediate. Suppose this pair were $P R Q$ and $Q S P$. If $\mathscr{S}_{2}$ denotes the quadrangle packing $\{P, Q, R, S,[P Q],[P R],[Q R]$, $[P S],[Q S],[P Q R],[P Q S]\}$ then by Lemma 6 we obtain

$$
\mathscr{A}\left(\mathscr{S}_{2}\right) / \Delta^{*} \geqq|P R||R Q|+|P S||S Q|
$$

and hence

$$
\begin{aligned}
& \mathscr{A}\left(\mathscr{S}_{2}\right) / \Delta^{*}-\mathscr{P}\left(\mathscr{S}_{2}\right)+2 \\
& \quad \geqq|P R||R Q|+|P S||S Q|-|P R|-|Q R|-|P S|-|Q S|+2 \\
& \quad=(|P R|-1)(|Q R|-1)+(|P S|-1)(|Q S|-1) \geqq 0 .
\end{aligned}
$$

Similarly, if the pair of intermediate angles were $R Q S$ and $S P R$ then we would have

$$
\mathscr{A}\left(\mathscr{S}_{2}\right) / \Delta^{*} \geqq|P R||P S|+|Q R||Q S|
$$

and again (8) holds.

Now, if we delete $[P Q]$ and the two triangles $[P Q R]$ and $[P Q S]$ from $\mathscr{S}$ we obtain a finite packing $\mathscr{S}_{1}$ for which $a_{0}\left(\mathscr{S}_{1}\right)=a_{0}(\mathscr{S})$, $a_{1}\left(\mathscr{S}_{1}\right)<a_{1}(\mathscr{S}), a_{2}\left(\mathscr{S}_{1}\right)<a_{2}(\mathscr{S}), \chi\left(\mathscr{S}_{1}\right)=\chi(\mathscr{S})-1, \mathscr{A}\left(\mathscr{S}_{1}\right)=\mathscr{A}(\mathscr{S})-$ $\mathscr{A}(P Q R)-\mathscr{A}(P R S), \mathscr{P}\left(\mathscr{S}_{1}\right)=\mathscr{P}(\mathscr{S})+|P R|+|R Q|+|P S|+|S Q|$, and $a\left(\mathscr{S}_{1}\right)<a(\mathscr{S})$.

By the minimality assumptions on $\mathscr{S},(3)$ must hold for $\mathscr{S}_{1}$. Thus

$$
\begin{aligned}
a_{0}(\mathscr{S})=a_{0}\left(\mathscr{S}_{1}\right) \leqq & \left.\mathscr{A}\left(\mathscr{S}_{1}\right) / 2\right\lrcorner^{*}+\mathscr{P}\left(\mathscr{S}_{1}\right) / 2+\chi\left(\mathscr{S}_{1}\right) \\
= & \mathscr{A}(\mathscr{S}) / 2 \Delta^{*}+\mathscr{P}(\mathscr{S}) / 2+\chi(\mathscr{S}) \\
& -(\mathscr{A}(P Q R)+\mathscr{A}(P Q S)) / 2 \Delta^{*} \\
& +(|P R|+|R Q|+|P S|+|S Q|) / 2+1 \\
\leqq & \mathscr{A}(\mathscr{S}) / 2 \Delta^{*}+\mathscr{P}(\mathscr{S}) / 2+\chi(\mathscr{S})
\end{aligned}
$$

by (8). This contradicts the assumption that (3) fails for $\mathscr{S}$ and the theorem is proved.

9. Tight packings. Theorem 4 suggests the following

Definition. The slackness of a finite packing $\mathscr{S}$ is defined as the nonnegative number

$$
\tau(\mathscr{S})=\mathscr{A}(\mathscr{S}) /\left(2 \Delta^{*}\right)+\mathscr{P}(\mathscr{S}) / 2+\chi(\mathscr{S})-a_{0}(\mathscr{S}) .
$$

The finite packing $\mathscr{S}$ is said to be tight if its slackness is zero.

Going carefully through the steps of the proofs of Theorem 4 and the preceding lemmas we obtain 
Theorem 5. A finite packing $\mathscr{S}$ is tight if and only if there is a triangulation $\mathscr{S}^{\prime}$ of $|\mathscr{S}|$ using the same vertex set as $\mathscr{S}$ such that all edges are length 1 and all triangles of $\mathscr{S}^{\prime}$ are equilateral of side length 1 and of area $\Delta^{*}$.

COROLLARY. If there are only two equilateral triangles over any basis then a connected packing without nonbounding edges is tight if and only if its vertices belong to a critical lattice and its boundary edges are of length 1 (for terminology see [8]).

As an application of Theorem 4 we can prove the result first shown by N. Oler [4] that the vertices of an equilateral triangle of sidelength 1 and area $\Delta^{*}$ generate a critical lattice and vice versa (for the original statement and proof see [8]).

The packing inequality (3) suggests a metric treatment of the FPSC's $\mathscr{S}$ by associating with them the nonnegative dimensionless packing constant

$$
\tau_{0}(\mathscr{S})=\mathscr{A}(\mathscr{S}) /\left(2 \Delta^{*} \mu_{0}(\mathscr{S})^{2}\right)+\mathscr{P}(\mathscr{S}) /\left(2 \mu_{0}(\mathscr{S})\right)+a_{2}(\mathscr{S})-a_{1}(\mathscr{S})
$$

where $\mu_{0}(\mathscr{S})$ is the minimum of the length of the edges of $\mathscr{S} \cdot$ This packing constant is invariant under homotheties. It is independent of scale changes for the Minkowski distance. It is zero if and only if $\mathscr{S}$ is tight for the Minkowski distance obtained from the given one by a scale change in the ratio $\mu_{0}(\mathscr{S}): 1$.

\section{REFERENCES}

1. W. Blaschke, Vorlesungen über Integral Geometrie 2 Heft, Teubner 1937 p. 109-110.

2. J. H. Folkman and R. L. Graham, A packing inequality for compact convex subsets of the plane, Canad. Math. Bull., 12 (1969), 745-752.

3. N. Oler, An inequality in the geometry of numbers, Acta Mathematica, 105 (1961), $19-48$.

4. —, A finite packing problem, Canad. Math. Bull., 4 (1961), 153-155.

5. - The slackness of finite packings in $E_{2}$, Amer. Math. Monthly, 69 (1964), $511-514$.

6. — Packings with Lacunae, Duke Math. J., 33 (1966), 141-144.

7. C. A. Rogers, Packing and Covering, Cambridge Tracts in Mathematics and Mathematical Physics, 54 (1964).

8. - The closest packing of convex two-dimensional domains, Acta Mathematica, 86 (1951), 309-321.

9. H. Zassenhaus, Modern developments in the geometry of numbers, Bull. Amer. Math. Soc., 67 (1961), 427-439.

Received March 18, 1971.

Bell Telephone Laboratories, Incorporated

AND

The OHio State University 



\title{
PACIFIC JOURNAL OF MATHEMATICS
}

\author{
EDITORS
}

H. SAMELSON

Stanford University

Stanford, California 94305

C. R. HOBBY

University of Washington

Seattle, Washington 98105

\section{J. DugundJI}

Department of Mathematics

University of Southern California

Los Angeles, California 90007

RICHARD ARENS

University of California

Los Angeles, California 90024

\section{ASSOCIATE EDITORS}
E. F. BeCKeNBACH
B. H. NeUmanN
F. WoLF
K. YoSHIDA

\section{SUPPORTING INSTITUTIONS}

\author{
UNIVERSITY OF BRITISH COLUMBIA \\ CALIFORNIA INSTITUTE OF TECHNOLOGY \\ UNIVERSITY OF CALIFORNIA \\ MONTANA STATE UNIVERSITY \\ UNIVERSITY OF NEVADA \\ NEW MEXICO STATE UNIVERSITY \\ OREGON STATE UNIVERSITY \\ UNIVERSITY OF OREGON \\ OSAKA UNIVERSITY
}

\author{
UNIVERSITY OF SOUTHERN CALIFORNIA \\ STANFORD UNIVERSITY \\ UNIVERSITY OF TOKYO \\ UNIVERSITY OF UTAH \\ WASHINGTON STATE UNIVERSITY \\ UNIVERSITY OF WASHINGTON

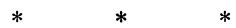 \\ AMERICAN MATHEMATICAL SOCIETY \\ NAVAL WEAPONS CENTER
}

The Supporting Institutions listed above contribute to the cost of publication of this Journal, but they are not owners or publishers and have no responsibility for its content or policies.

Mathematical papers intended for publication in the Pacific Journal of Mathematics should be in typed form or offset-reproduced, (not dittoed), double spaced with large margins. Underline Greek letters in red, German in green, and script in blue. The first paragraph or two must be capable of being used separately as a synopsis of the entire paper. The editorial "we" must not be used in the synopsis, and items of the bibliography should not be cited there unless absolutely necessary, in which case they must be identified by author and Journal, rather than by item number. Manuscripts, in duplicate if possible, may be sent to any one of the four editors. Please classify according to the scheme of Math. Rev. Index to Vol. 39. All other communications to the editors should be addressed to the managing editor, Richard Arens, University of California, Los Angeles, California, 90024.

50 reprints are provided free for each article; additional copies may be obtained at cost in multiples of 50 .

The Pacific Journal of Mathematics is published monthly. Effective with Volume 16 the price per volume (3 numbers) is $\$ 8.00$; single issues, $\$ 3.00$. Special price for current issues to individual faculty members of supporting institutions and to individual members of the American Mathematical Society: $\$ 4.00$ per volume; single issues $\$ 1.50$. Back numbers are available.

Subscriptions, orders for back numbers, and changes of address should be sent to Pacific Journal of Mathematics, 103 Highland Boulevard, Berkeley, California, 94708.

PUBLISHED BY PACIFIC JOURNAL OF MATHEMATICS, A NON-PROFIT CORPORATION

Printed at Kokusai Bunken Insatsusha (International Academic Printing Co., Ltd.), 270, 3-chome Totsuka-cho, Shinjuku-ku, Tokyo 160, Japan. 


\section{Pacific Journal of Mathematics}

\section{Vol. 41, No. $3 \quad$ BadMonth, 1972}

George E. Andrews, Two theorems of Gauss and allied identities proved arithmetically.................................. 563

Stefan Bergman, On pseudo-conformal mappings of circular domains . . . . 579

Beverly L. Brechner, On the non-monotony of dimension ............ 587

Richard Anthony Brualdi and John H. Mason, Transversal matroids and Hall's theorem .................................... 601

Philip Throop Church and James Timourian, Differentiable maps with

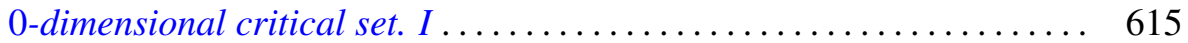

John H. E. Cohn, Squares in some recurrent sequences ............. 631

Robert S. Cunningham, Edgar Andrews Rutter and Darrell R. Turnidge, Rings of quotients of endomorphism rings of projective modules ......

Eldon Dyer and S. Eilenberg, An adjunction theorem for locally equiconnected spaces................................... 669

Michael W. Evans, On commutative P. P. rings................. 687

Ronald Lewis Graham, Hans Sylvain Witsenhausen and Hans Zassenhaus, On tightest packings in the Minkowski plane ..................

Stanley P. Gudder, Partial algebraic structures associated with

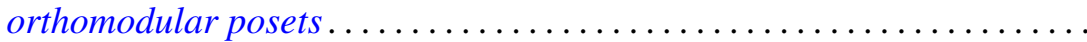

Karl Edwin Gustafson and Gunter Lumer, Multiplicative perturbation of

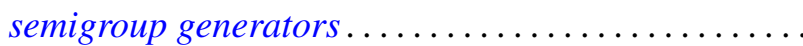

Kurt Kreith and Curtis Clyde Travis, Jr., Oscillation criteria for selfadjoint elliptic equations...

Lawrence Louis Larmore, Twisted cohomology theories and the single obstruction to lifting...........................

Jorge Martinez, Tensor products of partially ordered groups . . .

Robert Alan Morris, The inflation-restriction theorem for Amitsur cohomology ...

Leo Sario and Cecilia Wang, The class of $(p, q)$-biharmonic functions ...

Manda Butchi Suryanarayana, On multidimensional integral equations of

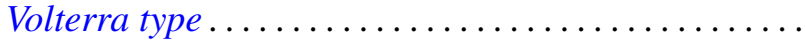

